\title{
Radar Scattering From Coated Perfect Conduc- tors: Application to the Semi-Infinite Cone and Use of Exact Eikonal
}

\author{
H. Überall ${ }^{1}$
}

\author{
Contribution From the Conductron Corporation, Ann Arbor, Mich.
}

(Received November 26, 1963)

\begin{abstract}
An approximation method for calculating the diffraction of electromagnetic waves from perfect conductors coated with weakly scattering material is developed, which consists essentially of a combination of the physical optics approximation (for the perfect conductors) with the eikonal method of Saxon and Schiff (for the coating). A Born-type expression for the scattering amplitude may be obtained as a simplified version of the more general results, but is shown to be of qualitative value only. Numerical results are presented for the case of nose-on backscattering from a dielectrically coated semi-infinite perfectly conducting cone. For this special example, we alternatively use an exact eikonal Green's function, thus extending the results of the Saxon-Schiff eikonal method to include small cone opening angles and arbitrary complex dielectric constant and permeability of the coating. In an appendix, the same problem is treated by the straightforward physical-optics approximation.
\end{abstract}

\section{Introduction}

Some interest has arisen recently in the scattering of electromagnetic waves from coated bodies [Hiatt, Siegel, and Weil, 1960; Weston and Hemenger, 1962]. The following paper is a contribution to this subject. We consider a situation where perfect conductors and weak scatterers (complex dielectric constant and permeability with magnitudes near unity) are present simultaneously, and develop a method, based on the application of Green's theorem and combining the usual Kirchhoff method (generalized to the vector case by Sommerfeld and by Stratton and Chu) with the eikonal method [Saxon and Schiff, 1957; Brown, 1959; Überall, 1962] for calculating the electromagnetic field scattered from such a system of obstacles. The general method is outlined in section 2 and carried as far as possible, although applications of $(21)$ to specific examples still require, as in the Kirchhoff approximation, a judicious choice of physical-optics fields. Section 3 applies the method to scattering from a semi-infinite perfectly conducting an uniformly coated cone not a completely general example, since complications arising from shadows are avoided. Here, Born-type fields are also obtained as simplified forms of the results, but are shown numerically to give only qualitative approximations. In section 4, the problem of the semi-infinite coated cone is treated again, this time using an exact eikonal function rather than one corresponding to straight line propagation; the results are thereby extended to small cone opening angles and arbitrary scattering: power of the coating. Graphs of the radar cross sections are presented. In appendix 1, physical-optics fields of coated, perfectly conducting planes are worked out. Appendix 2 treats the problem of the coated cone by the straightforward physical-optics approximation.

\section{General Formulation}

The essence of our method will be an application of Green's theorem to Maxwell's equations, in order to express the scattered field at infinity by the values of the field integrated over all boundary and discontinuity surfaces, as well as over the volumes of the scatterers coating the perfect conductors. Analogously to the customary physical-optics (or Kirchhoff) approxima-

\footnotetext{
${ }^{1}$ Also at the Harrison M. Randall Laboratory of Physics, The University of Michigan, Ann Arbor, Mich.

The work described in this paper was in part the result of work done for the Air Force under Subcontract to the Conductron Corporation as a part of the program being carried out under AVCO Corporation Prime Contract AF 04(694)-264.
} 
tion, the fields in the integrals may then be replaced by those present on the surface and in the coating of an infinitely large, perfectly conducting coated plane; as usual, this should give good results if the radius of curvature of the bodies is large compared to the wavelength. The use of the eikonal rather than the free-space Green's function is expected to improve the results over those of Born approximation similarly as in the diffraction from an isolated weak scatterer [Überall, 1962].

With a harmonic time dependence $\exp (-i k t)$ of the fields (setting $c=1, \epsilon_{0}=\mu_{0}=1$ ), Maxwell's equations become

$$
\begin{array}{ll}
\boldsymbol{\nabla} \times \mathbf{E}=i k \mu \mathbf{H}, & \boldsymbol{\nabla} \cdot \epsilon^{\prime} \mathbf{E}=0, \\
\boldsymbol{\nabla} \times \mathbf{H}=-i k \epsilon^{\prime} \mathbf{E}, & \boldsymbol{\nabla} \cdot \mu \mathbf{H}=0 .
\end{array}
$$

The dielectric constant is complex:

$$
\epsilon^{\prime}=\epsilon(1+i \nu), \quad \nu=\sigma / k \epsilon,
$$

and so may be the permeability $\mu$. Taking the curl of the first equation (1b), one has

$$
\boldsymbol{\nabla} \times \boldsymbol{\nabla} \times \mathbf{H}=\epsilon^{\prime-1}\left(\boldsymbol{\nabla} \epsilon^{\prime}\right) \times(\boldsymbol{\nabla} \times \mathbf{H})+K^{2} \mathbf{H},
$$

a wave equation with propagation constant

$$
K=k\left(\epsilon^{\prime} \mu\right)^{1 / 2} .
$$

To apply Green's theorem, the eikonal Green's function of Saxon and Schiff [1957] will be used:

with eikonal phase

$$
F\left(\mathbf{r}, \mathbf{r}^{\prime}\right)=F\left(\mathbf{r}^{\prime}, \mathbf{r}\right)=-(4 \pi \rho)^{-1} e^{i k \rho} e^{i D}
$$

$$
D\left(\mathbf{r}, \mathbf{r}^{\prime}\right)=\int_{0}^{\rho}\left[K\left(\mathbf{r}^{\prime}+\hat{\rho} s\right)-k\right] d s
$$

( $\rho=\mathbf{r}-\mathbf{r}^{\prime}, \hat{\rho}=\boldsymbol{\rho} / \rho$ ), corresponding to propagation along straight line paths. This implies that we shall always consider situations where, besides perfect conductors, only weakly scattering bodies (with magnitudes of $\epsilon^{\prime}, \mu$ not much different from unity) are thought to be present. We can then introduce a dyadic

$$
\mathscr{J}\left(\mathbf{r}, \mathbf{r}^{\prime}\right)=\mathscr{I} F\left(\mathbf{r}, \mathbf{r}^{\prime}\right)
$$

with $\mathscr{I}$ the unit dyadic, satisfying the wave equation

$$
\boldsymbol{\nabla} \times \boldsymbol{\nabla} \times \mathscr{J}=-\mathscr{I}\left[\delta\left(\mathbf{r}-\mathbf{r}^{\prime}\right)-(\boldsymbol{\nabla} S)^{2} F+i F \rho^{2} \boldsymbol{\nabla} \cdot\left(\rho^{-2} \boldsymbol{\nabla} S\right)\right]+\boldsymbol{\nabla} \boldsymbol{\nabla} F,
$$

where $S=D+k \rho$. Now, we shall use the vector Green's theorem [Stratton, 1941]

$$
\int_{S} \mathbf{n} \cdot[\mathbf{B} \times \boldsymbol{\nabla} \times \mathbf{A}-\mathbf{A} \times \boldsymbol{\nabla} \times \mathbf{B}] d A=\int_{V}[\mathbf{A} \cdot \boldsymbol{\nabla} \times \boldsymbol{\nabla} \times \mathbf{B}-\mathbf{B} \cdot \boldsymbol{\nabla} \times \boldsymbol{\nabla} \times \boldsymbol{A}] d \tau
$$

and identify

$$
\mathbf{A} \rightarrow \mathbf{H}, \mathbf{B} \rightarrow \mathscr{f} \cdot \mathbf{a}=F \mathbf{a},
$$

a being an arbitrary constant vector. The expressions (3) and (7) will then be introduced in (8). Before that, however, we have to specify the surfaces $S$ and volumes $V$ over which the integrals in (8) will be taken. Since the bodies have sharp surfaces which will give rise to reflections, we shall apply (8) separately to all regions $V_{i}$ of space separated from the other regions by closed surfaces of discontinuity. These are: (1) the material surfaces across which there 
are abrupt changes of $\epsilon^{\prime}, \mu$ as well as of the fields, due to the boundary conditions

$$
\begin{aligned}
\hat{n} \cdot\left(\epsilon_{1} \mathbf{E}_{1}-\epsilon_{2} \mathbf{E}_{2}\right)=\omega, & \hat{n} \times\left(\mathbf{E}_{1}-\mathbf{E}_{2}\right)=0, \\
\hat{n} \cdot\left(\mu_{1} \mathbf{H}_{1}-\mu_{2} \mathbf{H}_{2}\right)=0, & \hat{n} \times\left(\mathbf{H}_{1}-\mathbf{H}_{2}\right)=\mathbf{K},
\end{aligned}
$$

with $\hat{n}$ the surface normal, $\omega$ and $\boldsymbol{K}$ being surface charge and current density (the latter nonvanishing only for perfect conductors; in the interiors of those the fields themselves vanish); and further (2) the boundary surfaces of the "shadows" of the material bodies which would be produced by a light source in $\mathbf{r}^{\prime}$. The reason for this is that the $S$ appearing in (7) has discontinuous derivatives across these surfaces, and it may even be discontinuous itself when the shadow surface coincides with a material surface. After application of Green's theorem, we shall let $\mathbf{r}^{\prime}$ go to infinity; the shadow "cones" will accordingly tend to cylinders. The boundaries between $V_{i}$ and $V_{k}$ shall be considered as double surfaces, i.e., $S_{i k}$ bounding $V_{i}$, with normal vector $\mathbf{n}_{i}$ pointing into $V_{k}$, and $S_{k i}$ bounding $V_{k}$, with normal $\mathbf{n}_{k}$ pointing into $V_{i}$. Green's theorem applied to all $V_{i}$, and all the results added together then leads to:

$$
\begin{aligned}
\sum_{i k} \int_{S_{i k}} \mathbf{n}_{i} \cdot[F \mathbf{a} \times \boldsymbol{\nabla} \times \mathbf{H}-\mathbf{H} \times((\boldsymbol{\nabla} F) \times \mathbf{a})] d S=-\mathbf{a} \cdot \mathbf{H}^{\prime} & +\mathbf{a} \cdot \sum_{i} \int_{V_{i}}\left\{F \mathbf{H}\left[(\boldsymbol{\nabla} S)^{2}-i \rho^{2} \boldsymbol{\nabla} \cdot\left(\rho^{-2} \boldsymbol{\nabla} S\right)-K^{2}\right]\right. \\
& \left.+\mathbf{H} \cdot \boldsymbol{\nabla} \boldsymbol{\nabla} F-F \boldsymbol{\epsilon}^{\prime-1}\left(\boldsymbol{\nabla} \boldsymbol{\epsilon}^{\prime}\right) \times \boldsymbol{\nabla} \times \mathbf{H}\right\} d \tau,
\end{aligned}
$$

where we write for simplicity: $\mathbf{H}(\mathbf{r})=\mathbf{H}, \mathbf{H}\left(\mathbf{r}^{\prime}\right)=\mathbf{H}^{\prime}$. Next, we split $\sum_{i k}$ into those contributions which together add up to that from the surface bordering infinity, $S_{\infty}$, plus the sum $\sum_{i k}^{\prime}$ over the remaining boundary surfaces. Using the asymptotic form of the fields,

$$
\mathbf{H}(\mathbf{r})=\mathbf{H}_{0} e^{i k \mathbf{r} \cdot \mathbf{n}_{0}}+\mathbf{A}\left(\mathbf{k}_{0}, \mathbf{k}\right) r^{-1} e^{i k r}
$$

with $\mathbf{n}_{0} \cdot \mathbf{H}_{0}=0, \mathbf{n}_{0}$ being a unit vector along the direction of incidence of the radiation, and $\mathbf{k}=k \mathbf{r} / r, \mathbf{k}_{0}=k \mathbf{n}_{0}$, we find

$$
\int_{S_{\infty}} \mathbf{n}_{\infty} \cdot\left[F \mathbf{a} \times \boldsymbol{\nabla} \times \mathbf{H}-\mathbf{H} \times\left(\left(\boldsymbol{\nabla} F^{\prime}\right) \times \mathbf{a}\right)\right] d S=-\mathbf{a} \cdot \mathbf{H}_{0} e^{i\left[\mathbf{k}_{0} \cdot \mathbf{r}^{\prime}+\delta_{0}\left(\mathbf{r}^{\prime}\right)\right]},
$$

where

$$
\delta_{0}(\mathbf{r})=\int_{0}^{\infty}\left[K\left(\mathbf{r}-\mathbf{n}_{0} s\right)-k\right] d s .
$$

The limiting expression of $F$,

$$
\lim _{r \rightarrow \infty} F\left(\mathbf{r}, \mathbf{r}^{\prime}\right)=-(4 \pi r)^{-1} e^{i\left(k r-\mathbf{k} \cdot \mathbf{r}^{\prime}\right)} e^{i \delta+\left(\mathbf{r}^{\prime}\right)}
$$

has also been used, with

$$
\delta_{+}\left(\mathbf{r}^{\prime}\right)=\lim _{r \rightarrow \infty} D\left(\mathbf{r}, \mathbf{r}^{\prime}\right)=\int_{0}^{\infty}[K(\mathbf{r}+\mathbf{n} s)-k] d s .
$$

Equation (13) represents the plane incoming wave in (12) which is modified only in the exact forward direction $\mathbf{n}_{0}$ by the phase shift $\delta_{0}$, as pointed out earlier [Überall, 1962]. Extracting further the vector a from the remaining surface integral by the use of some vector identities [Stratton, 1941], we obtain

$$
\begin{aligned}
\mathbf{H}^{\prime}=\mathbf{H}_{0} e^{i\left(\mathbf{k}_{0} \cdot \mathbf{r}^{\prime}+\delta_{0}^{\prime}\right)}+\sum_{i k}{ }_{i k} \int_{S_{i k}} d S\left[\left(\mathbf{n}_{i} \times \mathbf{H}\right) \times \boldsymbol{\nabla} F+F \mathbf{n}_{i} \times(\boldsymbol{\nabla} \times \mathbf{H})\right] \\
\quad+\sum_{i} \int_{V_{i}} d \tau\left\{F \mathbf{H}\left[(\boldsymbol{\nabla} S)^{2}-K^{2}-i \rho^{2} \boldsymbol{\nabla} \cdot\left(\rho^{-2} \boldsymbol{\nabla} S\right)\right]+\mathbf{H} \cdot \boldsymbol{\nabla} \boldsymbol{\nabla} F-F \epsilon^{\prime-1}\left(\boldsymbol{\nabla} \epsilon^{\prime}\right) \times \boldsymbol{\nabla} \times \mathbf{H}\right\} .
\end{aligned}
$$

This can further be simplified if we consider on the one hand a sum $\sum_{i}$ ' over the volumes of all material bodies plus possible free space volumes enclosed by bodies (all these are finite volumes), 
and on the other hand a sum $\sum_{i}^{\prime \prime}$ over all shadow volumes in free space that reach to $S_{\infty}$; in them, $\boldsymbol{\nabla}_{\epsilon^{\prime}}=0$. The remaining volumes are just free space reaching to $S_{\infty}$, with $\boldsymbol{\nabla} \epsilon^{\prime}=0, K=k$, $S=k \rho$, and thus in these volume integrals, the square bracket vanishes. When we finally in (17) let $\mathbf{r}^{\prime}$ tend to infinity along $\mathbf{n}=\mathbf{r}^{\prime} / r^{\prime}$ in order to obtain the asymptotic form (12) and to read off the scattering amplitude $A$, we have to remember that such a limit may be taken before the integrals are actually performed only if they are "restricted," i.e., if the integration goes over a finite volume or surface. In order to achieve this, we want to transform some of the integrals containing $\mathbf{H} \cdot \boldsymbol{\nabla} \nabla F$ into surface integrals. Consider the expression

$$
\sum_{i k} \int_{S_{i k}} d S \mathbf{n}_{i} \cdot[(\boldsymbol{\nabla} \times \mathbf{H}) \times \boldsymbol{\nabla}(\mathbf{a} \cdot \boldsymbol{\nabla} F)],
$$

integrated over $S_{\infty}$, the shadow surfaces in free space reaching up to $S_{\infty}$, and the surfaces separating the material from all free space that reaches to $S_{\infty}$. By application of Gauss' theorem and Maxwell's equations in free space, we may transform this into

$$
k^{2} \mathbf{a} \cdot \sum_{i} \int_{V_{i}} d \tau \mathbf{H} \cdot \nabla \nabla F
$$

over all volumes in free space that reach to $S_{\infty}$. However, the contribution to (18) from $S_{\infty}$ vanishes due to the boundary conditions (12) and (15); using vector identities, it becomes further

$$
\sum_{i \vec{k}^{\prime \prime}}^{\prime \prime} \int_{S_{i k}} d S\left[\mathbf{n}_{i} \times(\boldsymbol{\nabla} \times \mathbf{H})\right] \cdot \boldsymbol{\nabla}(\mathbf{a} \cdot \boldsymbol{\nabla} F),
$$

where $\sum_{i k}^{\prime \prime}$ includes the shadow surfaces in free space reaching up to $S_{\infty}$ (but not $S_{\infty}$ itself), and the surfaces separating the material from all free space that reaches up to $S_{\infty}$. An example for all such surfaces and volumes will be presented in the figure of the following section. Altogether, we have for our general results the final expression

$$
\begin{array}{rl}
\mathbf{H}^{\prime}=\mathbf{H}_{0} e^{i\left(\mathbf{k}_{0} \cdot \mathbf{r}^{\prime}+\delta_{0^{\prime}}\right)}+\sum_{i k}{ }^{\prime} \int_{S_{i k}} & d S\left[\left(\mathbf{n}_{i} \times \mathbf{H}\right) \times \boldsymbol{\nabla} F+F \mathbf{n}_{i} \times(\boldsymbol{\nabla} \times \mathbf{H})\right] \\
& +k^{-2} \sum_{i k}^{\prime \prime} \int_{S_{i k}} d S\left[\mathbf{n}_{i} \times(\boldsymbol{\nabla} \times \mathbf{H})\right] \cdot \boldsymbol{\nabla} \boldsymbol{\nabla} F \\
& +\sum_{i}^{\prime} \int_{V_{i}} d \tau\left\{F \mathbf{H}\left[(\boldsymbol{\nabla} S)^{2}-K^{2}-i \rho^{2} \boldsymbol{\nabla} \cdot\left(\rho^{-2} \boldsymbol{\nabla} S\right)\right]\right. \\
& \left.+\mathbf{H} \cdot \boldsymbol{\nabla} \boldsymbol{\nabla} F-F \epsilon^{\prime-1}\left(\boldsymbol{\nabla} \epsilon^{\prime}\right) \times \boldsymbol{\nabla} \times \mathbf{H}\right\} \\
& \quad+\sum_{i}^{\prime \prime} \int_{V_{i}} d \tau F \mathbf{H}\left[(\boldsymbol{\nabla} S)^{2}-k^{2}-i \rho^{2} \boldsymbol{\nabla} \cdot\left(\rho^{-2} \boldsymbol{\nabla} S\right)\right] .
\end{array}
$$

Just as in the case of the usual Kirchhoff theory, the general procedure stops here with the advice to insert physical-optics fields into the integrals, which have to be chosen specifically for fitting: each individual situation. For example, in the case of a perfect conductor covered with a uniform coating, one might use the known fields present on the surfaces and in the coating of a coated perfectly conducting infinite plane, for the illuminated side of the object; zero in the region shadowed by the conductor. Some general remarks may, however, still be made: (a) All integrals over the volume of the perfect conductors and their shadows, as well as over the surfaces bounding them, vanish due to the factor exp $(i D)$ (or $\exp \left(i \grave{\delta}_{+}\right)$) which contains $K$, its imaginary part being positive and $\sim \sigma^{1 / 2}$, tending to $+\infty$ for perfect conductors. (Note that in the shadow, the line of integration of $D$ or $\delta_{+}$still passes through the conductor.) (b) The 
Born approximation is valid when the eikonal phases are small, and may be obtained from (21) by taking the limit $\delta_{0} \rightarrow 0, \delta_{+} \rightarrow 0$. Since $\delta_{+}$generates the discontinuity surfaces of type 2 , these surfaces will be absent in Born approximation. Indeed, Green's theorem would in this case have been applied to the material surfaces and volumes only, excluding the volumes of the perfect conductors, and only those integrations will appear in the Born equivalent of (21). (c) Guided by the Born approximation, the integrals in $\sum_{i k}^{\prime}, \sum_{i k}^{\prime \prime}$ over the shadow surfaces reaching to $S_{\infty}$ could quite possibly be dropped altogethrr if physical-optics fields cannot easily be found for them. This would also be suggested physically by the fact that shadows of finite bodies do not reach to infinity, but are washed out over distances $\geq R^{2} / \lambda$, where $R$ is the dimension of the diffracting obstacle. (d) The remaining surface integrals, as well as the integrals in $\sum_{i}^{\prime}$, are all restricted, and the limit $r^{\prime} \rightarrow \infty$, equivalent to (15), may be taken in them immediately. (e) The remarks under (c) can also be applied to $\sum_{i}^{\prime \prime}$. However, these integrals may be transformed into integrals over restricted regions by methods outlined earlier [Saxon and Schiff, 1957; Úberall, 1962]. (f) Our suggested method to obtain approximate solutions out of (21) is not the iteration method of Saxon and Schiff [1957], but is comparable to the more intuitive Kirchhoff approximation [Sommerfeld, 1959]. The conditions of applicability of the method are then no longer the clear-cut ones of the iteration [Überall, 1962, (24), (28)], e.g., $k R>>1$, but become more obscure. Generally speaking, it is known that Kirchhoff's method works best when the wavelength is small compared to the longest dimension of the obstacle. (g) Due to the boundary condition on the surface of a perfect conductor

$$
\hat{n} \times \mathbf{E}=0
$$

[from (10a), owing to the vanishing of the fields in perfect conductors], and from Maxwell's equation (1b, first equation), the second integral and the second term in the first integral of (21) vanish on these surfaces. This is the essence of all the methods using the formula of Stratton and Chu [Stratton, 1941; Kerr, 1951] for the calculation of the scattered fields.

\section{Application to a Semi-Infinite, Perfectly Conducting Coated Cone}

The method outlined in the preceding section will now be applied to a specific example, and many of the general ideas may become clearer in the application. The example chosen, namely diffraction of electromagnetic waves by a semi-infinite perfectly conducting cone coated with a homogeneous weakly scattering material of uniform thickness, represents, however, not the most general case, since it avoids all the complications arising from the shadows. We shall start out with a finite coated cone, as shown in figure 1. The sums over integration surfaces and volumes needed in (21) are here as follows:

$$
\begin{aligned}
& \sum_{i k}^{\prime} \text { contains } S_{01}, S_{10}, S_{12}, S_{21}, S_{23}, S_{32}, S_{14}, S_{41}, S_{04}, S_{40}, S_{34}, S_{43} ; \\
& \sum_{i k}^{\prime \prime} \text { contains } S_{01}, S_{04}, S_{40}, S_{41}, S_{43}, S_{34}, S_{32} ; \\
& \sum_{i}^{\prime} \text { contains } V_{1}, V_{2} ; \\
& \sum_{i}^{\prime \prime} \text { contains } V_{3}, V_{4} .
\end{aligned}
$$

Since $F=0$ inside the perfect conductor $V_{2}$ and its shadow $V_{3}$, the volumes $V_{2}$ and $V_{3}$ and the surfaces $S_{2 i}, S_{3}$ may be dropped. The semi-infinite cone may be considered as a limiting case of figure 1 , with $r_{0} \rightarrow \infty$. The cone basis, as will be demonstrated later, and the shadows are then removed to infinity and give no contribution, so that all surfaces and volumes with indices 


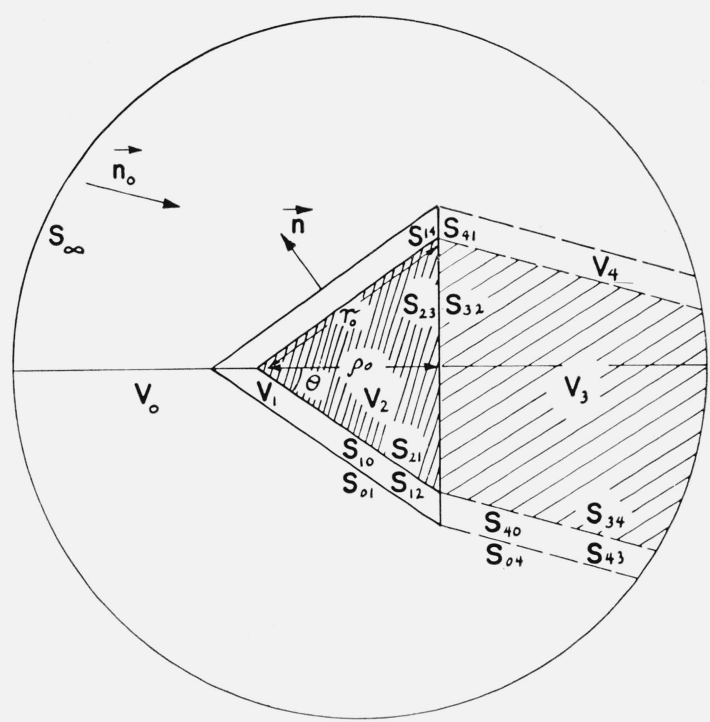

Figure 1. Material and shadow volumes and surfaces for the case of a finite coated cone.

3, 4 may also be dropped; this leaves us with:

$$
\begin{aligned}
& \sum_{i k}^{\prime} \text { contains } S_{01}, S_{10}, S_{12} ; \\
& \sum_{i k}^{\prime \prime} \text { contains } S_{01} ; \\
& \sum_{i}^{\prime} \text { contains } V_{1} ; \\
& \sum_{i}^{\prime \prime} \text { gives no contribution. }
\end{aligned}
$$

We also assume homogeneous coating, thus $\nabla \epsilon_{1}{ }^{\prime}=0$, and further on the conducting surface $S_{12}, \mathbf{n}_{i} \times \boldsymbol{\nabla} \times \mathbf{H}=0$. If we call

$$
\mathbf{H}_{s c}=\mathbf{H}-\mathbf{H}_{0} e^{i \mathbf{k}_{0} \cdot \mathbf{r}},
$$

then, since we cannot consider any scattering in the exact forward direction,

$$
\begin{aligned}
\mathbf{H}_{s c}^{\prime}=\int_{S_{01}+S_{10}} d S\left[\left(\mathbf{n}_{i} \times \mathbf{H}\right) \times \boldsymbol{\nabla} F+F \mathbf{n}_{i} \times(\boldsymbol{\nabla} \times \mathbf{H})\right] & \\
& +k^{-2} \int_{S_{01}} d S\left[\mathbf{n}_{i} \times(\boldsymbol{\nabla} \times \mathbf{H})\right] \cdot \boldsymbol{\nabla}(\boldsymbol{\nabla} F)+\int_{S_{12}}\left(\mathbf{n}_{i} \times \mathbf{H}\right) \times \boldsymbol{\nabla} F d S \\
& \quad+\int_{V_{1}} d \tau\left\{F \mathbf{H}\left[(\boldsymbol{\nabla} S)^{2}-K^{2}-i \rho^{2} \boldsymbol{\nabla} \cdot\left(\rho^{-2} \boldsymbol{\nabla} S\right)\right]+\mathbf{H} \cdot \boldsymbol{\nabla} \boldsymbol{\nabla} F\right\} .
\end{aligned}
$$

The square bracket in the last integral may be rewritten as

$$
-e^{-i D} \nabla^{2} e^{i D}-(K-k)^{2}+2 i(K-k) \rho^{-1} .
$$

If the limit $r_{0} \rightarrow \infty$ (see fig. 1) is not yet taken in (23), then all integrals are "restricted," and we may let $\mathbf{r}^{\prime}$ go to infinity in $F\left(\mathbf{r}, \mathbf{r}^{\prime}\right)$, so that (23) represents the scattered far field, related to the scattering amplitude $\mathbf{A}\left(\mathbf{k}_{0}, \mathbf{k}\right)$ by (12).

Let us also specialize to the case of backscattering, so that $\mathbf{k} \rightarrow-\mathbf{k}_{0}, \delta_{+} \rightarrow \delta_{0}$. Further, we introduce one common unit normal vector $\mathbf{n}$ to all cone surfaces, assumed to point away from 
the body of the cone. The result is

$$
\begin{aligned}
\mathbf{H}_{b s c}^{\prime}(\infty)=-\left(4 \pi r^{\prime}\right)^{-1} e^{i k r^{\prime}}\left\{-\int_{S_{01}} d S\left[(\mathbf{n} \times \mathbf{H}) \times \boldsymbol{\nabla} e^{i\left(\mathbf{k}_{0} \cdot \mathbf{r}+\delta_{0}\right)}+e^{i\left(\mathbf{k}_{0} \cdot \mathbf{r}+\delta_{0}\right)} \mathbf{n} \times(\boldsymbol{\nabla} \times \mathbf{H})\right.\right. \\
\left.+k^{-2}[\mathbf{n} \times(\boldsymbol{\nabla} \times \mathbf{H})] \cdot \boldsymbol{\nabla} \boldsymbol{\nabla} e^{i\left(\mathbf{k}_{0} \cdot \mathbf{r}+\delta_{0}\right)}\right]+\int_{S_{10}} d S\left[(\mathbf{n} \times \mathbf{H}) \times \boldsymbol{\nabla} e^{i\left(\mathbf{k}_{0} \cdot \mathbf{r}+\delta_{0}\right)}\right. \\
\left.+e^{i\left(\mathbf{k}_{0} \cdot \mathbf{r}+\delta_{0}\right)} \mathbf{n} \times(\boldsymbol{\nabla} \times \mathbf{H})\right]-\int_{S_{12}} d S(\mathbf{n} \times \mathbf{H}) \times \boldsymbol{\nabla} e^{i\left(\mathbf{k}_{0} \cdot \mathbf{r}+\delta_{0}\right)} \\
\\
\quad-\int_{V_{1}} d \tau\left[e^{i\left(\mathbf{k}_{0} \cdot \mathbf{r}+\delta_{0}\right)} \mathbf{H}\left[(K-k)^{2}+e^{-i \delta_{0}} \nabla^{2} e^{\left.i \delta_{0}\right]}-\mathbf{H} \cdot \boldsymbol{\nabla} \nabla e^{i\left(\mathbf{k}_{0} \cdot \mathbf{r}+\delta_{0}\right)}\right]\right\}
\end{aligned}
$$

The Born-Kirchhoff approximation result would be obtained from this by letting $\delta_{0} \rightarrow 0$, leading to a greatly simplified equation. A partially simplified result, which we shall refer to as "Born-type" approximation, may be obtained by keeping $\delta_{0}$ in the rapidly varying exponentials, but neglecting derivatives of $\delta_{0}$. This leads to

$$
\begin{aligned}
\mathbf{H}_{b s c}^{\prime}(\infty) \cong-i k\left(4 \pi r^{\prime}\right)^{-1} e^{i k r^{\prime}} & \left\{\int_{S_{01}} d S e^{i \mathbf{k}_{0} \cdot \mathbf{r}}\left[\mathbf{n} \times \mathbf{E}-\mathbf{n}_{0} \mathbf{n}_{0} \cdot(\mathbf{n} \times \mathbf{E})\right]\right. \\
-\int_{S_{10}} d S e^{i \mathbf{k}_{0} \cdot \mathbf{r}} \epsilon^{\prime}(\mathbf{n} \times \mathbf{E})- & \int_{S_{12}} d S e^{i\left(\mathbf{k}_{0} \cdot \mathbf{r}+\delta_{0}\right)}(\mathbf{n} \times \mathbf{H}) \times \mathbf{n}_{0} \\
& \left.+i k \int_{V_{1}} d \tau e^{i\left(\mathbf{k}_{0} \cdot \mathbf{r}+\delta_{0}\right)}\left[\mathbf{H}(N-1)^{2}+\mathbf{n}_{0} \mathbf{n}_{0} \cdot \mathbf{H}\right]\right\},
\end{aligned}
$$

using an index of refraction $N=\left(\epsilon^{\prime} \mu\right)^{\frac{1}{2}}=K / k$.

At this point, the physical-optics approximation will be made. For the fields on the surfaces of the cone and its coating, and in the volume of the coating, we shall insert the exact fields which are present on the surface and in the coating of an infinite perfectly conducting plane, coated uniformly by a homogeneous weak scatterer of thickness $\delta$. Appendix 1 outlines how this field is obtained. This is expected to lead to good results if the radius of curvature is larger than the wavelength of the incident radiation. Obviously this is not true in a region with dimensions of less than a wavelength around the tip of the cone. It is known, however, that the scattered field may be thought to be produced by currents which are set up in a region stretching at least a wavelength away from the tip [V. H. Weston, private communication]. In any case, the physical-optics approximation for scattering from a perfectly conducting, uncoated semi-infinite cone leads to results [Siegel and Alperin, 1952] which are in extremely good agreement with the exact values [Hansen and Schiff, 1948; Siegel et al., 1955]; one may therefore expect this to be true to some extent also for the coated cone.

Since the normal vector $\mathbf{n}$ of appendix 1 is not constant as one moves over the surface of the cone, we should introduce another reference direction. Using a unit vector e pointing from the tip into the cone along its axis (and we shall in the following always put the origin into the tip of the perfectly conducting cone; $\mathbf{r}_{p} \cdot \mathbf{n}=0$ then still holds), let us decompose the incident field:

$$
\mathbf{H}_{0}=H_{\perp} \mathbf{e} \times \mathbf{n}_{0}+H_{\|} \mathbf{n}_{0} \times\left(\mathbf{e} \times \mathbf{n}_{0}\right) .
$$

One then finds

$$
\begin{aligned}
& a_{0}=\left[\left(\mathbf{n}_{0} \cdot \mathbf{e n} \mathbf{n}_{0} \cdot \mathbf{n}-\mathbf{n} \cdot \mathbf{e}\right) H_{\perp}+\mathbf{n} \cdot\left(\mathbf{e} \times \mathbf{n}_{0}\right) H_{\|}\right]\left[1-\left(\mathbf{n}_{0} \cdot \mathbf{n}\right)^{2}\right]^{-1}, \\
& b_{0}=\left[\mathbf{n} \cdot\left(\mathbf{e} \times \mathbf{n}_{0}\right) H_{\perp}-\left(\mathbf{n}_{0} \cdot \mathbf{e n} \mathbf{n}_{0} \cdot \mathbf{n}-\mathbf{n} \cdot \mathbf{e}\right) H_{\|}\right]\left[1-\left(\mathbf{n}_{0} \cdot \mathbf{n}\right)^{2}\right]^{-1} .
\end{aligned}
$$

Further, since the Saxon-Schiff method applies to weak scatterers and assumes straight-line propagation of the rays, ef (5b), we shall for the sake of consistency and simplicity make the 
same approximation in the physical-optics fields of appendix 1: set $\mathbf{n}_{0}^{\prime} \cong \mathbf{n}_{0}, \mathbf{n}_{1}^{\prime} \cong \mathbf{n}_{1}$. We obtain:

$$
\begin{aligned}
& a_{1} \cong e^{2 i \alpha} \frac{N \cos N \alpha-i \mu \sin N \alpha}{N \cos N \alpha+i \mu \sin N \alpha} a_{0}, \\
& a_{0}^{\prime} \cong e^{i \alpha} \frac{N \mu}{N \cos N \alpha+i \mu \sin N \alpha} a_{0}, \\
& b_{1} \cong-e^{2 i \alpha} \frac{N \cos N \alpha-i \mu \sin N \alpha}{N \cos N \alpha+i \mu \sin N \alpha} b_{0}, \\
& b_{0}^{\prime} \cong e^{i \alpha} \frac{N \mu}{N \cos N \alpha+i \mu \sin N \alpha} b_{0},
\end{aligned}
$$

with

$$
\alpha=\mathbf{n}_{0} \cdot \mathbf{n} a, a=k \delta .
$$

The integrals in (24) will be evaluated for a simple case only, namely for nose-on backscattering. Then $\mathbf{n}_{0}=\mathbf{e}$, and the problem becomes very symmetric. For the surface integrals we use polar coordinates $r, \vartheta, \varphi(\vartheta$, half the opening angle of the cone, is constant) and have $d S=\sin \vartheta r d r d \varphi$. For integrals over $S_{01}$ or $S_{10}$, we shift the origin into the tip of the coating by the transformation $\mathbf{r}^{\prime}=\mathbf{r}+\mathbf{e} \delta \csc \vartheta$. For the volume integrals, we use cylindrical coordinates $\rho, \varphi, z$, and have

$$
\int d \tau=\int_{0}^{\rho_{0}} \rho d \rho \int_{\rho \cot \vartheta-\delta \csc \vartheta}^{\rho \cot \vartheta} d z \int_{0}^{2 \pi} d \varphi
$$

also

$$
\begin{gathered}
\delta_{0}=k(N-1)(z+\delta \csc \vartheta-\rho \cot \vartheta), \\
\boldsymbol{\nabla} \delta_{0}=-\mathbf{n} k(N-1) \csc \vartheta .
\end{gathered}
$$

It is the surface integral over $S_{12}$ in (24) which will, both in the limits $\delta \rightarrow 0$ (or $a \rightarrow 0$ ) and $N \rightarrow 1$, go over into the physical-optics result for a bare cone [Siegel and Alperin, 1952; Siegel, Crispin, and Schensted, 1955]

$$
H_{b s c}^{\prime}(\infty)=\frac{i e^{i k r^{\prime}}}{4 k r^{\prime}} H_{0} \tan ^{2} \vartheta
$$

(actually, in the limit $a \rightarrow 0$, we shall obtain this result multiplied by a factor $4 N(N+1)^{-2}$, which is, however, $\cong 1$ in second order in $(N-1)$ ), whereas all the other integrals go to zero. In this integral, we also have $\delta_{0}=k(N-1) \delta \csc \vartheta$, and generally, $\mathbf{n}_{0} \cdot \mathbf{n}=-\sin \vartheta$.

Using (31b), we may rewrite (24) in the form

$$
\mathbf{H}_{b s c}^{\prime}(\infty)=-i k\left(4 \pi r^{\prime}\right)^{-1} e^{i k r^{\prime}}\left\{\mathbf{J}_{1}+\left(1-\epsilon^{\prime}\right) \mathbf{J}_{2}-\epsilon^{\prime} \mathbf{J}_{3}+\mathbf{J}_{4}+(N-1) \csc \vartheta\left[\mathbf{L}_{1}+\mathbf{L}_{2}+\mathbf{L}_{3}+\mathbf{L}_{4}\right]\right\},
$$

where terms of orders $(N-1)^{2}$ have been neglected, but terms $\sim(N-1)$ were kept. Use of the Born-type approximation (25) would have given (33) without the term containing the square bracket (thus leading to inconsistencies in powers of $(N-1)$ ). The individual terms are:

$$
\begin{aligned}
& \mathbf{J}_{1}=\int_{S_{12}} d S \mathbf{n}_{0} \times(\mathbf{n} \times \mathbf{H}) e^{i\left(\mathbf{k}_{0} \cdot \mathbf{r}+\delta_{0}\right)}, \\
& \mathbf{J}_{2}=\int_{S_{01}} d S \mathbf{n}_{0} \times\left[(\mathbf{n} \times \mathbf{E}) \times \mathbf{n}_{0}\right] e^{i \mathbf{k}_{0} \cdot \mathbf{r}}, \\
& \mathbf{J}_{3}=\mathbf{n}_{0} \int_{S_{01}} d S \mathbf{n}_{0} \cdot(\mathbf{n} \times \mathbf{E}) e^{i \mathbf{k}_{0} \cdot \mathbf{r}}, \\
& \mathbf{J}_{4}=i k \mathbf{n}_{0} \int_{V_{1}} d \tau \mathbf{n}_{0} \cdot \mathbf{H} e^{i\left(\mathbf{k}_{0} \cdot \mathbf{r}+\delta_{0}\right)},
\end{aligned}
$$




$$
\begin{aligned}
& \mathbf{L}_{1}=\int_{S_{01}} d S \mathbf{n} \times(\mathbf{n} \times \mathbf{H}) e^{i \mathbf{k}_{0} \cdot \mathbf{r}}, \\
& \mathbf{L}_{2}=\int_{S_{12}} d S(\mathbf{n} \times \mathbf{H}) \times \mathbf{n} e^{i\left(\mathbf{k}_{0} \cdot \mathbf{r}+\delta_{0}\right)}, \\
& \mathbf{L}_{3}=-i k \int_{V_{1}} d \tau \mathbf{n} \mathbf{H} \cdot \mathbf{n}_{0} e^{i\left(\mathbf{k}_{0} \cdot \mathbf{r}+\delta_{0}\right)}, \\
& \mathbf{L}_{4}=-i k \mathbf{n}_{0} \int_{V_{1}} d \tau \mathbf{H} \cdot \mathbf{n} e^{i\left(\mathbf{k}_{0} \cdot \mathbf{r}+\delta_{0}\right)} .
\end{aligned}
$$

Since for nose-on scattering there is azimuthal symmetry, we may take $H_{\|}=0 . \quad$ Equations (27) then become by a limiting procedure:

$$
a_{0}=H_{0} \cos \varphi / \cos \vartheta, \quad b_{0}=H_{0} \sin \varphi / \cos \vartheta .
$$

In terms of these quantities, the physical-optics fields needed in (34) to (39), always using the straight-line approximation, are from (A12) to (A14), (28), (29), and using Maxwell's equations:

a. On $S_{12}$ :

$$
\begin{aligned}
& \mathbf{n}_{0} \times(\mathbf{n} \times \mathbf{H})=-2 N^{2} \Delta^{-1} e^{i a \mathbf{n}_{0} \cdot \mathbf{n}} \mathbf{n}_{0} \cdot \mathbf{n}\left[a_{0} \mathbf{n}_{0} \times \mathbf{n}+b_{0} \mathbf{n}_{0} \times\left(\mathbf{n} \times \mathbf{n}_{0}\right)\right] e^{i N \mathbf{k}_{0} \cdot \mathbf{r}_{p}}, \\
& \mathbf{n} \times(\mathbf{n} \times \mathbf{H})=-2 N^{2} \Delta^{-1} e^{i a \mathbf{n}_{0} \cdot \mathbf{n}}\left[a_{0} \mathbf{n}_{0} \times \mathbf{n}+b_{0}\left(\mathbf{n}_{0} \cdot \mathbf{n}\right) \mathbf{n} \times\left(\mathbf{n} \times \mathbf{n}_{0}\right)\right] e^{i N \mathbf{k}_{0} \cdot \mathbf{r}_{p}} .
\end{aligned}
$$

b. On $S_{01}$ :

$$
\begin{aligned}
& \mathbf{n}_{0} \times\left[(\mathbf{n} \times \mathbf{E}) \times \mathbf{n}_{0}\right]=2 i \mu \Delta^{-1} \mathbf{n}_{0} \cdot \mathbf{n} \sin \left(N a \mathbf{n}_{0} \cdot \mathbf{n}\right)\left[a_{0} \mathbf{n}_{0} \times \mathbf{n}+b_{0} \mathbf{n}_{0} \times\left(\mathbf{n} \times \mathbf{n}_{0}\right)\right] e^{i \mathbf{k}_{0} \cdot \mathbf{r}_{c}}, \\
& (\mathbf{n} \times \mathbf{H}) \times \mathbf{n}=2 N \Delta^{-1} \cos \left(N a \mathbf{n}_{0} \cdot \mathbf{n}\right)\left[a_{0} \mathbf{n}_{0} \times \mathbf{n}+b_{0}\left(\mathbf{n}_{0} \cdot \mathbf{n}\right) \mathbf{n} \times\left(\mathbf{n} \times \mathbf{n}_{0}\right)\right] e^{i \mathbf{k}_{0} \cdot \mathbf{r}_{c}},
\end{aligned}
$$

c. In $V_{1}$ :

$\mathbf{H}=N^{2} \Delta^{-1} e^{i a \mathbf{n}_{0} \cdot \mathbf{n}}\left\{a_{0} \mathbf{n}_{0} \times \mathbf{n}+b_{0} \mathbf{n}_{0} \times\left(\mathbf{n} \times \mathbf{n}_{0}\right)+\left[a_{0} \mathbf{n}_{0} \times \mathbf{n}\right.\right.$

where

$$
\left.\left.-b_{0}\left(\left(\mathbf{n}_{0} \cdot \mathbf{n}\right) \mathbf{n} \times\left(\mathbf{n}_{0} \times \mathbf{n}\right)+\mathbf{n}\left(1-\left(\mathbf{n}_{0} \cdot \mathbf{n}\right)^{2}\right)\right)\right] e^{-2 i N k\left(\mathbf{n}_{0} \cdot \mathbf{n}\right) \mathbf{n} \cdot \mathbf{r}}\right\} e^{i N \mathbf{k}_{0} \cdot \mathbf{r}},
$$

$$
\Delta=N \cos \left(N a \mathbf{n}_{0} \cdot \mathbf{n}\right)+i \mu \sin \left(\operatorname{Nan} \mathbf{n}_{0} \cdot \mathbf{n}\right) .
$$

With these fields, the integrals (34) to (39) are evaluated in the limit $r_{0} \rightarrow \infty$ or $\rho \rightarrow \infty$; in this case, there is no contribution from the base of the cone which moves away to infinity; "tip scattering" alone remains. (In the nose-on situation, there is formally an infinite contribution from the base as $r_{0} \rightarrow \infty$; physically, it must vanish due to the finite conductivity of air, and the zero result may also be obtained by starting out with nonaxial incidence and taking the limit of nose-on incidence afterwards. $)^{2}$ The result is:

with

$$
\mathbf{H}_{b s c}^{\prime}(\infty)=i\left(4 k r^{\prime}\right)^{-1} e^{i k r} \mathbf{H}_{0} \tan ^{2} \vartheta \cdot S,
$$

$S=e^{i a[(N-1) \csc \vartheta-\sin \vartheta]}\left[\cos (N a \sin \vartheta)-i \mu N^{-1} \sin (N a \sin \vartheta)\right]^{-1}$

$$
\begin{aligned}
& \times\left\{1+\left(1-\epsilon^{\prime}\right) i \mu N^{-1} \sin (N a \sin \vartheta) e^{-i a(N \csc \vartheta+\cos \vartheta \cot \vartheta)}\right. \\
& +(N-1) \csc \vartheta\left[\frac{1}{2}(\sin \vartheta+\csc \vartheta)\left(1-\cos (N a \sin \vartheta) e^{-i a(N \csc \vartheta+\cos \vartheta \cot \vartheta)}\right)\right. \\
& \left.\left.\quad-\sin \vartheta(N+1)^{-2}\left(1-e^{-2 i N a \cos \vartheta \cot \vartheta}\right)\right]\right\} .
\end{aligned}
$$

${ }^{2}$ All the "longitudinal" integrals (proportional to $\mathbf{n}_{0}$ ) vanish for nose-on incidence. 


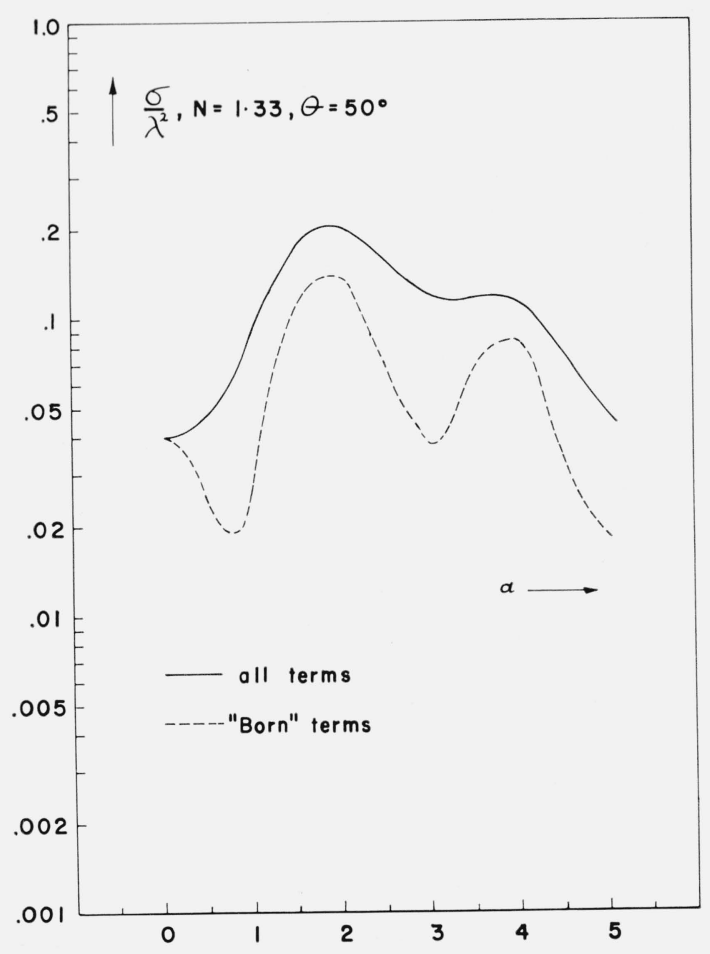

Figure 2. Radar cross section of a semi-infinite, perfectly conducting dielectrically coated cone (noseon incidence), plotted versus $a=\mathrm{k} \delta$ ( $\delta=$ thickness of coating) for various values of the refractive index $\mathrm{N}$ of the coating and of the half opening angle $\theta$ of the cone.

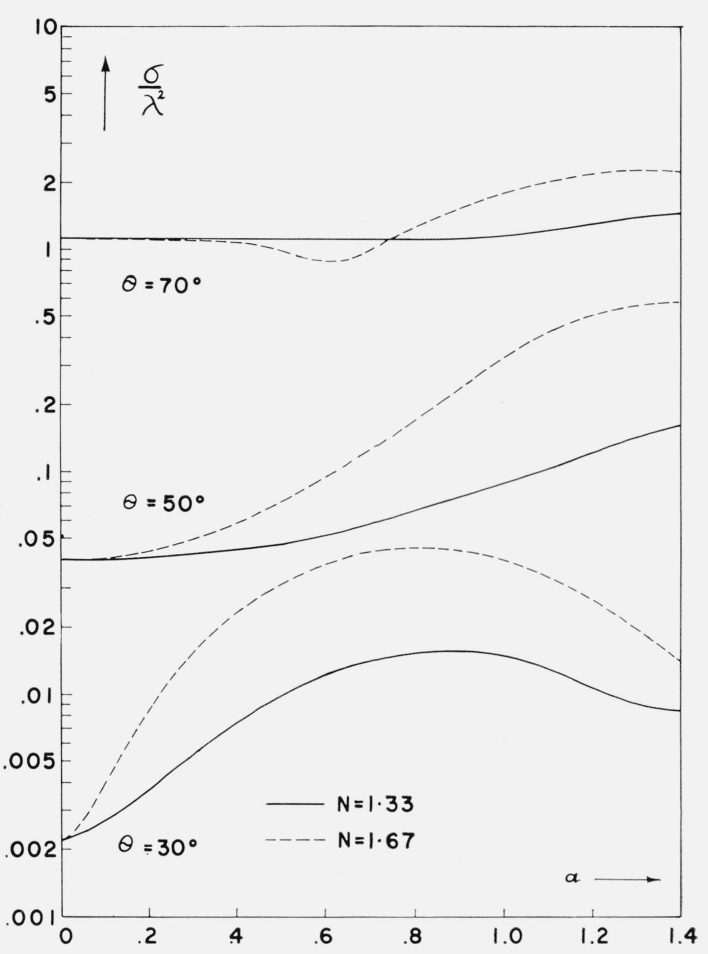

Figure 3. Comparison of radar cross section of the cone considered in figure 2 with results of $a$ Born-type approximation.

The radar cross section is obtained from

and we find

$$
\sigma=4 \pi r^{\prime 2}\left|\mathbf{H}_{b s c}^{\prime}(\infty)\right|^{2} \cdot\left|\mathbf{H}_{0}\right|^{-2},
$$

$$
\frac{\sigma}{\lambda^{2}}=\frac{\tan ^{4} \vartheta}{16 \pi}|S|^{2}
$$

where $\lambda$ is the wavelength of the incident radiation; (47) goes over into the result for the tip scattering from a bare perfectly conducting cone [Siegel et al., 1952 and 1955] in physicaloptics approximation if $S \rightarrow 1$. This is also the limit of (45) for $a \rightarrow 0$ or for $\epsilon^{\prime}, \mu \rightarrow 1$.

Values of $\sigma / \lambda^{2}$ are plotted versus $a$ in figure 2, for a dielectric coating with $N=1.33$ and 1.67, and for three cone half angles, $\vartheta=30^{\circ}, 50^{\circ}$, and $70^{\circ}$. The results are expected to be less accurate the smaller $\vartheta$ becomes, since then the basic assumption of straight-line approximation will be less accurate even for $N$ near unity.

The Born-type approximation for $S$ is obtained by dropping the term multiplied by $(N-1) \csc \vartheta$ in (45). It is clear that this is again less justifiable for smaller angles $\vartheta$. A comparison is made in figure 3, where $\sigma / \lambda^{2}$ is plotted versus $a$, with and without this approximation. One can see that the Born-type approximation reproduces no more than the general trend of the complete result in a qualitative fashion; it also exaggerates the diffraction minima, similarly as in Überall [1962]. For smaller angles, the discrepancies become even worse. 


\section{Use of Exact Eikonal for the Semi-Infinite Cone}

In the general situation of section 2, and in the application to the semi-infinite cone of section 3, we restricted ourselves to using an eikonal Green's function corresponding to straight-line propagation [Saxon and Schiff, 1957], for reasons of mathematical simplicity. This is equivalent to considering a weakly scattering coating (dielectric constant and permeability near unity) only; for small opening angles of the coated cone, assumption of straightline propagation will not be justified unless the scattering properties of the coating are extremely weak. The semi-infinite cone, however, is a sufficiently simple object so that one may use an exact eikonal Green's function which corresponds to the actual refraction of the rays in the coating material. This immediately removes the restriction to large cone angles and also to weakly scattering coating (cf, the remarks (f) of section 2). The integrals over the material boundaries and volume of the coating, in terms of which the far field is given after application of Green's theorem, are again evaluated by inserting the physical-optics field of an infinite, perfectly conducting coated plane.

As in section 2, we begin with the wave equation (3). The eikonal Green's function to be used is:

which satisfies

$$
F\left(\mathbf{r}, \mathbf{r}^{\prime}\right)=F\left(\mathbf{r}^{\prime}, \mathbf{r}\right)=-(4 \pi \rho)^{-1} e^{i S\left(\mathbf{r}, \mathbf{r}^{\prime}\right)},
$$

$$
\nabla^{2} F+(\boldsymbol{\nabla} S)^{2} F=\delta(\boldsymbol{\rho})+i F \rho^{2} \boldsymbol{\nabla} \cdot\left(\rho^{-2} \boldsymbol{\nabla} S\right),
$$

with $\boldsymbol{\rho}=\mathbf{r}-\mathbf{r}^{\prime}$. If the eikonal $S\left(\mathbf{r}, \mathbf{r}^{\prime}\right)$ satisfies certain limiting conditions [Brown, 1959], we obtain by application of the vector Green's theorem again the general equation (21), or for the coated cone, (23) for the scattered field at $\mathbf{r}^{\prime}$, where $S_{01}$ is the boundary just outside and $S_{10}$ that just inside the outer surface of the coating, $S_{12}$ the surface of the coating separating it from the perfectly conducting cone, $V_{1}$ the volume of the coating, and $\mathbf{n}_{i}$ the normal vectors of $S_{i k}$ pointing into $V_{k}$ or away from $V_{i}$. Later, a common normal vector $\mathbf{n}$ pointing out of the cone will be used.

The function $S\left(\mathbf{r}, \mathbf{r}^{\prime}\right)=S\left(\mathbf{r}^{\prime}, \mathbf{r}\right)$ will now be chosen, not as in 2 corresponding to straight-line propagation from $\mathbf{r}^{\prime}$ to $\mathbf{r}$ with WKB phase, but following a suggestion of Saxon [1957] as the classical action function satisfying

$$
\left[\nabla S\left(\mathbf{r}, \mathbf{r}^{\prime}\right)\right]^{2}=[K(\mathbf{r})]^{2},
$$

where $K$ is the propagation constant in the medium in which $\mathbf{r}$ is located. For an infinite, uniformly coated plane (in anticipation of the physical-optics approximation to be used later) [and also for nose-on incidence on the cone when $\mathbf{r}^{\prime}$ is removed to infinity (corresponding to the far field)], $S$ may be taken as

$$
S\left(\mathbf{r}, \mathbf{r}^{\prime}\right)=\left\{\begin{array}{l}
k \rho, \\
k \rho^{\prime \prime}+K \rho^{\prime}, \\
K \rho^{\prime \prime}+k \rho^{\prime}, \\
K \rho,
\end{array}\right.
$$

with $\boldsymbol{\rho}^{\prime}=\mathbf{r}-\mathbf{r}_{1}, \boldsymbol{\rho}^{\prime \prime}=\mathbf{r}_{1}-\mathbf{r}^{\prime}$, corresponding to (a) $\mathbf{r}, \mathbf{r}^{\prime}$ both in air, (b) $\mathbf{r}$ in the coating, $\mathbf{r}^{\prime}$ in air, (c) $\mathbf{r}$ in air, $\mathbf{r}^{\prime}$ in the coating, (d) $\mathbf{r}$, $\mathbf{r}^{\prime}$ both in the coating. The point $\mathbf{r}_{1}$, situated on the surface of the coating, is determined from the law of refraction:

$$
k \hat{\rho}^{\prime \prime} \times \mathbf{n}=K \hat{\rho}^{\prime} \times \mathbf{n}
$$

(for case (b)), with $\hat{\rho}^{\prime}=\boldsymbol{\rho}^{\prime} / \rho^{\prime}$, etc., and $\mathbf{n}$ the normal vector to the plane (or the cone) pointing into the air. It can be shown easily using (51) that (50a) to (50d) satisfies (50) as well as the necessary limiting conditions on $S$ [Brown, 1959]; we also used $\mathbf{r}_{1} \cdot \mathbf{n}=\delta$ where $\delta$ is the normal 
thickness of the coating (assuming always that the origin is situated on the conducting surface; in this case, we have on the surface of the conductor, $\mathbf{r} \equiv \mathbf{r}_{p}: \mathbf{n} \cdot \mathbf{r}_{p}=0$; on the surface of the coating, $\mathbf{r} \equiv \mathbf{r}_{c}: \mathbf{n} \cdot \mathbf{r}_{c}=\delta$, both for the plane and the cone).

To obtain the scattered far field in the direction $\mathbf{N}$ from(23), we remove $\mathbf{r}^{\prime} \equiv \mathbf{N} r^{\prime}$ to infinity and obtain

with $\mathbf{k}=k \mathbf{N}$;

$$
\lim _{r^{\prime} \rightarrow \infty} F\left(\mathbf{r}, \mathbf{r}^{\prime}\right)=-\left(4 \pi r^{\prime}\right)^{-1} e^{i k r} e^{-i \mathbf{k} \cdot \mathbf{r}^{\prime}+i \delta+(\mathbf{r})}
$$

$$
\delta_{+}(\mathbf{r})=K \rho^{\prime}+\mathbf{k} \cdot \boldsymbol{\rho}^{\prime}
$$

if $\mathbf{r}$ lies in the coating, and $\delta_{+}(\mathbf{r}) \equiv 0$ if $\mathbf{r}$ lies in air (this is also the value of $\delta_{+}$in general if the Born approximation, corresponding to $S=k \rho$, had been used).

Considering now only backscattering of the radiation, i.e., $\mathbf{N} \rightarrow-\mathbf{n}_{0}$ where $\mathbf{n}_{0}=\mathbf{k}_{0} / k$ is the direction of incidence of the radiation (along the cone axis if nose-on incidence is considered), then $\delta_{+}(\mathbf{r})$ becomes $\rightarrow$

and (24) now reads:

$$
\delta_{0}(\mathbf{r})=K \rho^{\prime}-\mathbf{k}_{0} \cdot \boldsymbol{\rho}^{\prime},
$$

$$
\begin{aligned}
\mathbf{H}_{b s c}^{\prime}(\infty)=-\left(4 \pi r^{\prime}\right)^{-1} e^{i k r},\left\{-\int_{S_{01}} d S\left[(\mathbf{n} \times \mathbf{H}) \times \boldsymbol{\nabla} e^{i\left(\mathbf{k}_{0} \cdot \mathbf{r}+\delta_{0}\right)}+e^{i\left(\mathbf{k}_{0} \cdot \mathbf{r}+\delta_{0}\right)} \mathbf{n} \times(\boldsymbol{\nabla} \times \mathbf{H})\right.\right. \\
\left.+k^{-2}[\mathbf{n} \times(\boldsymbol{\nabla} \times \mathbf{H})] \cdot \boldsymbol{\nabla} \boldsymbol{\nabla} e^{i\left(\mathbf{k}_{0} \cdot \mathbf{r}+\delta_{0}\right)}\right]-\int_{S_{12}} d S(\mathbf{n} \times \mathbf{H}) \times \boldsymbol{\nabla} e^{i\left(\mathbf{k}_{0} \cdot \mathbf{r}+\delta_{0}\right)} \\
+\int_{S_{10}} d S\left[(\mathbf{n} \times \mathbf{H}) \times \boldsymbol{\nabla} e^{i\left(\mathbf{k}_{0} \cdot \mathbf{r}+\delta_{0}\right)}+e^{i\left(\mathbf{k}_{0} \cdot \mathbf{r}+\delta_{0}\right)} \mathbf{n} \times(\boldsymbol{\nabla} \times \mathbf{H})\right] \\
\left.-\int_{V_{1}} d \tau\left[i e^{i\left(\mathbf{k}_{0} \cdot \mathbf{r}+\delta_{0}\right)} \mathbf{H} \nabla^{2} \delta_{0}-\mathbf{H} \cdot \boldsymbol{\nabla} \boldsymbol{\nabla} e^{i\left(\mathbf{k}_{0} \cdot \mathbf{r}+\delta_{0}\right)}\right]\right\} .
\end{aligned}
$$

If we rewrite (54) in the form

we find, using (51),

$$
\delta_{0}(\mathbf{r})=g(a-k \mathbf{n} \cdot \mathbf{r})
$$

$$
g=\mathbf{n}_{0} \cdot \mathbf{n}+\left[N^{2}-1+\left(\mathbf{n}_{0} \cdot \mathbf{n}\right)^{2}\right]^{1 / 2}
$$

with $a=k \delta, N=K / k$, and the square root is defined to have a positive real part. Note that (53), (54), (56), and (57), as well as the following equations, may be derived using vector identities only (which are formally valid also for complex $K$ ), rather than geometrical considerations. We also obtain the following identities:

$$
\begin{gathered}
\hat{\rho}^{\prime} \cdot \mathbf{n}=-N^{-1}\left[N^{2}-1+\left(\mathbf{n}_{0} \cdot \mathbf{n}\right)^{2}\right]^{1 / 2}, \\
\rho^{\prime}=\left(\hat{\rho}^{\prime} \cdot \mathbf{n}\right)^{-1}(\mathbf{r} \cdot \mathbf{n}-\delta), \\
\hat{\rho}^{\prime} \cdot \mathbf{n}_{0}=N^{-1}\left\{1-\left(\mathbf{n}_{0} \cdot \mathbf{n}\right)^{2}-\mathbf{n}_{0} \cdot \mathbf{n}\left[N^{2}-1+\left(\mathbf{n}_{0} \cdot \mathbf{n}\right)^{2}\right]^{1 / 2}\right\} ;
\end{gathered}
$$

if we introduce the half-opening angle of the cone $\vartheta$, then

$$
\mathbf{n}_{0} \cdot \mathbf{n}=-\sin \vartheta
$$

Analogously to (33), the backscattered far field may be written as

$$
\mathbf{H}_{b s c}^{\prime}(\infty)=-i k\left(4 \pi r^{\prime}\right)^{-1} e^{i k r^{\prime}}\left\{\mathbf{J}_{1}+\left(1-\epsilon^{\prime}\right) \mathbf{J}_{2}-\epsilon^{\prime} \mathbf{J}_{3}+\mathbf{J}_{4}+g\left(\mathbf{L}_{1}+\mathbf{L}_{2}+\mathbf{L}_{3}+\mathbf{L}_{4}\right)+g^{2} \mathbf{M}\right\},
$$

with $\mathbf{J}_{i}, \mathbf{L}_{i}$ as before, and with

$$
\mathbf{M}=i k \int_{V_{1}} d_{\tau} \mathbf{n} \mathbf{H} \cdot \mathbf{n} e^{i\left(\mathbf{k}_{\theta} \cdot \mathbf{r}+\delta_{0}\right)} .
$$


The difference with (33) is now that $\delta_{0}$ and $g$ are changed; in particular, that $g$ [which earlier was given by $(N-1) \csc \vartheta]$ is now no longer considered as small compared with unity, so that a term $g^{2} \mathbf{M}$ was kept in (62); and that in (62), the exact physical-optics fields above a coated plane have to be used, rather than those simplified by the straight-line approximation, as in section 2. These fields have been worked out in appendix 1, and the quantities needed in the integrals of (62) are as follows:

(a) on $S_{12}\left(\mathbf{r}=\mathbf{r}_{p}\right)$ :

with

$$
\begin{aligned}
& \mathbf{n}_{0} \times(\mathbf{n} \times \mathbf{H})=2 \mu^{-1}\left[a_{0}^{\prime}\left(\mathbf{n}_{0} \cdot \mathbf{n}\right) \mathbf{n} \times \mathbf{n}_{0}+b_{0}^{\prime}\left(\hat{\boldsymbol{\rho}}^{\prime} \cdot \mathbf{n}\right) \mathbf{n}_{0} \times\left(\mathbf{n}_{0} \times \mathbf{n}\right)\right] e^{i \mathbf{k}_{0} \cdot \mathbf{r}_{p}}, \\
& (\mathbf{n} \times \mathbf{H}) \times \mathbf{n}=2 \mu^{-1}\left[a_{0}^{\prime} \mathbf{n}_{0} \times \mathbf{n}+b_{0}^{\prime}\left(\hat{\boldsymbol{\rho}}^{\prime} \cdot \mathbf{n}\right) \mathbf{n} \times\left(\mathbf{n} \times \mathbf{n}_{0}\right)\right] e^{i \mathbf{k}_{0} \cdot \mathbf{r}_{p}},
\end{aligned}
$$

where $\alpha=a \mathbf{n}_{0} \cdot \mathbf{n}$, and

$$
\begin{aligned}
& a_{0}^{\prime}=N \mu \mathbf{n}_{0} \cdot \mathbf{n} e^{i \alpha} \Delta_{a}^{-1} a_{0}, \\
& b_{0}^{\prime}=N \mu \mathbf{n}_{0} \cdot \mathbf{n} e^{i \alpha} \Delta_{b}^{-1} b_{0},
\end{aligned}
$$

$$
\begin{gathered}
\Delta_{a}=N \mathbf{n}_{0} \cdot \mathbf{n} \cos \alpha^{\prime}+i \mu \hat{\rho}^{\prime} \cdot \mathbf{n} \sin \alpha^{\prime}, \\
\Delta_{b}=N \hat{\rho}^{\prime} \cdot \mathbf{n} \cos \alpha^{\prime}+i \mu \mathbf{n}_{0} \cdot \mathbf{n} \sin \alpha^{\prime}, \\
\alpha^{\prime}=N a \hat{\rho}^{\prime} \cdot \mathbf{n} .
\end{gathered}
$$

(b) on $S_{01}\left(\mathbf{r}=\mathbf{r}_{c}=\mathbf{r}_{p}+\mathbf{n} \delta\right)$ :

$$
\begin{aligned}
& \mathbf{n}_{0} \times\left[(\mathbf{n} \times \mathbf{E}) \times \mathbf{n}_{0}\right]=\left(\mathbf{n}_{0} \cdot \mathbf{n}\right)\left[\mathbf{n}_{0} \times \mathbf{n} A_{-}+\mathbf{n}_{0} \times\left(\mathbf{n} \times \mathbf{n}_{0}\right) B_{+}\right] e^{i \mathbf{k}_{0} \cdot \mathbf{r}_{\nu}}, \\
& \mathbf{n} \times(\mathbf{n} \times \mathbf{H})=\left[\mathbf{n} \times \mathbf{n}_{0} A_{+}+\left(\mathbf{n}_{0} \cdot \mathbf{n}\right) \mathbf{n} \times\left(\mathbf{n}_{0} \times \mathbf{n}\right) B_{-}\right] e^{i \mathbf{k}_{0} \cdot \mathbf{r}_{p}},
\end{aligned}
$$

with

$$
\begin{aligned}
& A_{+}=2 N \mathbf{n}_{0} \cdot \mathbf{n} \cos \alpha^{\prime} e^{i \alpha} \Delta_{a}^{-1} a_{0}, \\
& A_{-}=2 i \mu \hat{\rho}^{\prime} \cdot \mathbf{n} \sin \alpha^{\prime} e^{i \alpha} \Delta_{a}^{-1} a_{0}, \\
& B_{+}=2 i \mu \mathbf{n}_{0} \cdot \mathbf{n} \sin \alpha^{\prime} e^{i \alpha} \Delta_{b}^{-1} b_{0}, \\
& B_{-}=2 N \hat{\rho}^{\prime} \cdot \mathbf{n} \cos \alpha^{\prime} e^{i \alpha} \Delta_{b}^{-1} b_{0},
\end{aligned}
$$

(c) in $V_{1}$ :

$$
\begin{aligned}
\mathbf{H}=\mu^{-1}\left\{a_{0}^{\prime} \mathbf{n}_{0} \times \mathbf{n}+b_{0}^{\prime} N\left[\mathbf{n}-\left(\hat{\rho}^{\prime} \cdot \mathbf{n}\right) \hat{\rho}^{\prime}\right]+\left[a_{0}^{\prime} \mathbf{n}_{0} \times \mathbf{n}-b_{0}^{\prime}\left[\left(\hat{\rho}^{\prime} \cdot \mathbf{n}\right) \mathbf{n} \times\left(\mathbf{n}_{0} \times \mathbf{n}\right)\right.\right.\right. \\
\left.\left.\left.+N \mathbf{n}\left(1-\left(\hat{\rho}^{\prime} \cdot \mathbf{n}\right)^{2}\right)\right]\right] e^{-2 i N k \hat{\rho} \cdot \mathbf{n n} \cdot \mathbf{r}}\right\} e^{i N k \hat{\rho}^{\prime} \cdot \mathbf{r}} .
\end{aligned}
$$

For nose-on scattering, $a_{0}$ and $b_{0}$ are those given by (40).

We then obtain for the integrals (for nose-on backscattering), keeping the tip-scattering contribution only:

$$
\begin{aligned}
& \mathbf{J}_{1}=\left(2 k^{2}\right)^{-1} \pi \mathbf{H}_{0} \tan ^{2} \vartheta e^{i a(w-2 \sin \vartheta)}\left\{N \sin \vartheta \Delta_{a}^{-1}+w \Delta_{b}^{-1}\right\}, \\
& \mathbf{J}_{2}=\left(2 k^{2} N\right)^{-1} \pi \mathbf{H}_{0} \tan ^{2} \vartheta i \mu \sin (a w) e^{-2 i a \csc \vartheta}\left\{w \Delta_{a}^{-1}+N \sin \vartheta \Delta_{b}^{-1}\right\}, \\
& \mathbf{J}_{3}=\mathbf{J}_{4}=0, \\
& \mathbf{L}_{1}=-\left(2 k^{2}\right)^{-1} \pi \mathbf{H}_{0} \tan ^{2} \vartheta \cos (a w) e^{-2 i a \csc \vartheta}\left\{N \Delta_{a}^{-1}+w \sin \vartheta \Delta_{b}^{-1}\right\}, \\
& \mathbf{L}_{2}=\left(2 k^{2}\right)^{-1} \pi \mathbf{H}_{0} \tan ^{2} \vartheta e^{i a(w-2 \sin \vartheta)}\left\{N \Delta_{a}^{-1}+w \sin \vartheta \Delta_{b}^{-1}\right\},
\end{aligned}
$$




$$
\begin{aligned}
& \mathbf{L}_{3}=-\left(8 k^{2} \Delta_{b}\right)^{-1} \pi \mathbf{H}_{0} \tan ^{2} \vartheta e^{i a(w-2 \sin \vartheta)}\left\{(w-\sin \vartheta) \cos ^{2} \vartheta\left[1-e^{-2 i a(w+\cos \vartheta \cot \vartheta)}\right]\right. \\
&\left.\times(w+\cos \vartheta \cot \vartheta)^{-1}+(w+\sin \vartheta) \sin \vartheta\left[1-e^{-i i a \cos \vartheta \cot \vartheta}\right]\right\},
\end{aligned}
$$

$\mathbf{L}_{4}=0$,

$$
\begin{aligned}
\mathbf{M}=\left(8 k^{2} \Delta_{b}\right)^{-1} \pi \mathbf{H}_{0} \tan ^{2} \vartheta e^{i a(w-2 \sin \vartheta)}\left\{\cos ^{2} \vartheta\right. & {\left[1-e^{-2 i a(w+\cos \vartheta \cot \vartheta)}\right] } \\
& \left.\times(w+\cos \vartheta \cot \vartheta)^{-1}-\sin \vartheta\left[1-e^{-2 i a \cos \vartheta \cot \vartheta}\right]\right\},
\end{aligned}
$$

with

$$
w=\left(N^{2}-\cos ^{2} \vartheta\right)^{1 / 2}
$$

As before, we write the backscattered far field in the form of (44), and obtain for the radar cross section $\sigma$ the expression of (47). Again, it goes over into the result for the tip scattering from a bare perfectly conducting cone in physical-optics approximation if $S \rightarrow 1$. This is indeed the case for $a \rightarrow 0$, and/or for $\epsilon^{\prime}, \mu \rightarrow 1$, in our expression for $S$ :

$$
\begin{gathered}
S=A \Delta_{a}^{-1}+B \Delta_{b}^{-1}, \\
A=\frac{1}{2} N\left\{e^{-2 i a \csc \vartheta}\left[(w-\sin \vartheta) \cos (a w)+i\left(1-\epsilon^{\prime-1}\right) w \sin (a w)\right]-w e^{i a(w-2 \sin \vartheta)}\right\}, \\
B=\frac{1}{2} \sin \vartheta\left\{e^{-2 i a \csc \vartheta}\left[w(w-\sin \vartheta) \cos (a w)+i N^{2}\left(1-\epsilon^{\prime-1}\right) \sin (a w)\right]\right. \\
\left.+e^{i a(w-2 \sin \vartheta)} w\left[\frac{1}{2}(w-\sin \vartheta)\left(1-e^{-2 i a \cos \vartheta \cot \vartheta}\right)-(w+\cos \vartheta \cot \vartheta)\right]\right\} .
\end{gathered}
$$

In the same notation, $\Delta_{a}$ and $\Delta_{b}$ become:

$$
\begin{aligned}
& \Delta_{a}=-N \sin \vartheta \cos (a w)+i \mu N^{-1} w \sin (a w), \\
& \Delta_{b}=-w \cos (a w)+i \mu \sin \vartheta \sin (a w) .
\end{aligned}
$$

If the limit of straight-line propagation is taken carefully, (80) can be shown to go over into the previous result, (45).

Numerical results ${ }^{3}$ are shown in figure 4 where $|S|^{2}$ is plotted versus $a$, for $a$ ranging from 0 to $1 \cdot 2$, with a cone half-opening angle $\theta=10^{\circ}$ and for a purely dielectric coating of real dielectric constant, $\epsilon^{\prime}=3.0$, and $\mu=1.0$. For such a small opening angle, the result is a rapidly varying function of $a=k \delta$, showing pronounced diffraction maxima and minima. If the expression (45) for $|S|^{2}$ had been used (which is not expected to be valid for small $\theta$, due to the breakdown of the straight-line approximation), we would have found results resembling those of figure 4 , but with the peaks vastly larger (by a factor $\sim 10$ ), of approximately the same height each, and with a much shallower minimum which, however, was at the same position as that in figure 4. For large angles, though, (45) is expected to be a better approximation to $(80)$.

\section{Appendix 1}

In this appendix, we derive the fields on the surface and in the coating of an infinite perfectly conducting plane, uniformly coated by a homogeneous material of thickness $\delta$ with arbitrary complex dielectric constant and permeability. If there is an incident field $\mathbf{E}_{0} \exp \left(i \mathbf{k}_{0} \cdot \mathbf{r}\right)$, 


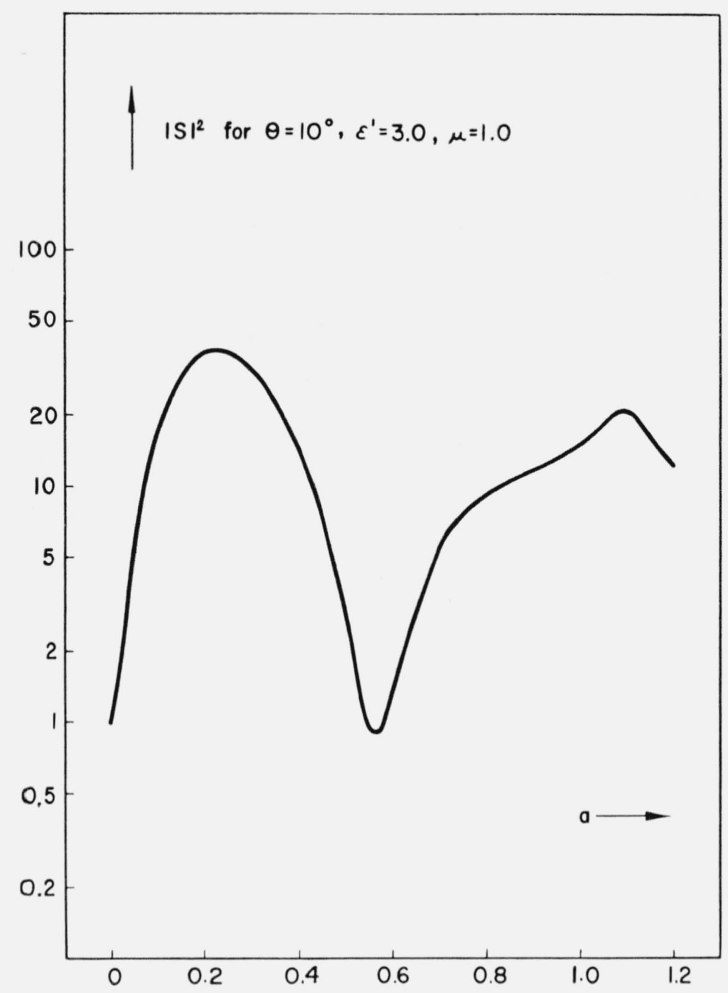

Figure 4. Radar cross section for axial incidence on a semi-infinite, perfectly conducting dielectrically coated cone, plotted versus $a=\mathrm{k} \delta$ (wave number $\mathrm{k}=2 \pi / \lambda$, thickness of coating $\delta$ ) for $\epsilon^{\prime}=3.0$, $\mu=1.0$ of the coating, and a half-opening angle of the cone $\theta=10^{\circ}$.

write the total field above the coating as

inside the coating as

$$
\mathbf{E}=\mathbf{E}_{0} e^{i \mathbf{k}_{0} \cdot \mathbf{r}}+\mathbf{E}_{1} e^{i \mathbf{k}_{1} \cdot \mathbf{r}},
$$

$$
\mathbf{E}^{\prime}=\mathbf{E}_{0}^{\prime} e^{i \mathbf{k}_{0}^{\prime} \cdot \mathbf{r}}+\mathbf{E}_{1}^{\prime} e^{i \mathbf{k}_{1}^{\prime} \cdot \mathbf{r}},
$$

where $\mathbf{k}_{0,1}=k \mathbf{n}_{0,1}, \mathbf{k}_{0,1}^{\prime}=k^{\prime} \mathbf{n}_{0,1}^{\prime}, k^{\prime} / k=N=\left(\epsilon^{\prime} \mu\right)^{1 / 2}$ (complex index of refraction), $\mathbf{n}_{0} \cdot \mathbf{E}_{0}=0, \ldots$ $\mathbf{n}_{1}^{\prime} \cdot \mathbf{E}_{1}^{\prime}=0$, and where the laws of reflection and refraction are

$$
\begin{array}{ll}
\mathbf{n}_{1}=\mathbf{n}_{0}-2 \mathbf{n}\left(\mathbf{n}_{0} \cdot \mathbf{n}\right), & \mathbf{n}_{1}^{\prime}=\mathbf{n}_{0}^{\prime}-2 \mathbf{n}\left(\mathbf{n}_{0}^{\prime} \cdot \mathbf{n}\right), \\
k \mathbf{n}_{0} \times \mathbf{n}=k^{\prime} \mathbf{n}_{0}^{\prime} \times \mathbf{n}, & k \mathbf{n}_{1} \times \mathbf{n}=k^{\prime} \mathbf{n}_{1}^{\prime} \times \mathbf{n},
\end{array}
$$

with $\mathbf{n}$ a unit normal vector pointing from the conductor into the coating. The boundary conditions (10) require on the outer surface of the coating $\left(\mathbf{r}=\mathbf{r}_{c}\right)$ :

$$
\mathbf{n} \times \mathbf{E}_{c}=\mathbf{n} \times \mathbf{E}_{c}^{\prime}, \quad \mathbf{n} \times \mathbf{H}_{c}=\mathbf{n} \times \mathbf{H}_{c}^{\prime},
$$

and on the surface of the perfectly conducting plane $\left(\mathbf{r}=\mathbf{r}_{p}=\mathbf{r}_{c}-\delta\right.$, where $\delta=\mathbf{n} \delta$; we also have $\mathbf{n} \cdot \mathbf{r}_{p}=0$ if the origin is put on the conducting plane):

$$
\mathbf{n} \times \mathbf{E}_{p}^{\prime}=0, \quad \mathbf{n} \cdot \mathbf{H}_{p}^{\prime}=0 ;
$$

the magnetic field is obtained from $\mathbf{E}$ always by the first equation (1a). Equation (A6) is satisfied with

$$
\mathbf{E}_{1}^{\prime}=2 \mathbf{n}\left(\mathbf{E}_{0}^{\prime} \cdot \mathbf{n}\right)-\mathbf{E}_{0}^{\prime} .
$$


At this point, the so-called "impedance boundary conditions" may easily be shown as a byproduct; these are

with

$$
\mathbf{n} \times\left(\mathbf{n} \times \mathbf{E}_{c}\right)=-\eta \mathbf{n} \times \mathbf{H}_{c},
$$

$$
\begin{gathered}
\eta=\eta_{\perp} \equiv-\frac{i \mu}{N} \frac{\tan \mathbf{k}_{0}^{\prime} \cdot \boldsymbol{\delta}}{\mathbf{n} \cdot \mathbf{n}_{0}^{\prime}}, \text { if } \mathbf{E}_{0}^{\prime} \perp \mathbf{n}, \mathbf{n}_{0}^{\prime} \\
\eta=\eta \| \equiv-\frac{i \mu}{N} \mathbf{n} \cdot \mathbf{n}_{0}^{\prime} \tan \mathbf{k}_{0}^{\prime} \cdot \boldsymbol{\delta}, \text { if } \mathbf{E}_{0}^{\prime} \text { is in the } \mathbf{n}, \mathbf{n}_{0}^{\prime} \text { plane, }
\end{gathered}
$$

and where for a strongly refracting or absorbing medium, $|N|>>1$,

$$
\eta_{\perp} \simeq \eta \|-i \mu N^{-1} \tan N k \delta,
$$

being polarization and angle independent.

The reflected and transmitted fields are obtained simply by satisfying (A5). If one writes

$$
\begin{aligned}
& \mathbf{E}_{0}=a_{0} \mathbf{n}_{0} \times\left(\mathbf{n} \times \mathbf{n}_{0}\right)+b_{0} \mathbf{n} \times \mathbf{n}_{0}, \\
& \mathbf{E}_{1}=a_{1} \mathbf{n}_{1} \times\left(\mathbf{n} \times \mathbf{n}_{1}\right)+b_{1} \mathbf{n} \times \mathbf{n}_{1}, \\
& \mathbf{E}_{0}^{\prime}=a_{0}^{\prime} \mathbf{n}_{0}^{\prime} \times\left(\mathbf{n} \times \mathbf{n}_{0}^{\prime}\right)+b_{0}^{\prime} \mathbf{n} \times \mathbf{n}_{0}^{\prime},
\end{aligned}
$$

then we obtain the results:

$$
\begin{aligned}
& a_{1}=e^{2 i \mathbf{k}_{0} \cdot \delta} \frac{\mathbf{n} \cdot \mathbf{n}_{0}+\eta \|}{\mathbf{n} \cdot \mathbf{n}_{0}-\eta \|} a_{0}, \\
& a_{0}^{\prime}=e^{i \mathbf{k}_{0} \cdot \delta} \frac{\mathbf{n} \cdot \mathbf{n}_{0}}{\mathbf{n} \cdot \mathbf{n}_{0}-\eta \|} \frac{\mu}{\cos \mathbf{k}_{0}^{\prime} \cdot \delta} a_{0} ; \\
& b_{1}=e^{2 i \mathbf{k}_{0} \cdot \delta} \frac{\mathbf{n} \cdot \mathbf{n}_{0}+\eta_{\perp}^{-1}}{\mathbf{n} \cdot \mathbf{n}_{0}-\eta_{\perp}^{-1}} b_{0}, \\
& b_{0}^{\prime}=e^{i \mathbf{k}_{0} \cdot \delta} \frac{\mathbf{n} \cdot \mathbf{n}_{0}}{\mathbf{n} \cdot \mathbf{n}_{0}-\eta_{\perp}^{-1}} \frac{N}{i \sin \mathbf{k}_{0}^{\prime} \cdot \boldsymbol{\delta}} b_{0} .
\end{aligned}
$$

\section{Appendix 2}

It may be of interest to see what results a straightforward physical-optics approximation would give for the scattering of radiation from a perfectly conducting cone with a homogeneous coating of uniform thickness. This calculation will turn out much simpler than the foregoing, since it does not incorporate the phase shifts, characteristic for the Saxon-Schiff approximation, which appear in the coating and on $S_{12}$.

Consider first a finite coated cone in free space. As before, we start from the vector Green's theorem, but use a conventional Green's function,

$$
G\left(\mathbf{r}, \mathbf{r}^{\prime}\right)=-(4 \pi \rho)^{-1} e^{i k \rho},
$$

with $\boldsymbol{\rho}=\mathbf{r}-\mathbf{r}^{\prime}$, instead of $F\left(\mathbf{r}, \mathbf{r}^{\prime}\right)$, and integrate over $V \equiv$ all of free space, bounded by $S_{01}$ (the outer surface of the coating), $S_{b}$ (the base surface of the cone), and $S_{\infty}$ (an infinite spherical surface with center at the origin which is again put into the tip of the conducting cone). In analogy to (17), we find

$$
\mathbf{H}^{\prime}=\mathbf{H}_{0} e^{i \mathbf{k}_{0} \cdot \mathbf{r}^{\prime}}+\int_{S_{b}+S_{01}} d S\left[\left(\mathbf{n}_{i} \times \mathbf{H}\right) \times \boldsymbol{\nabla} G+G \mathbf{n}_{i} \times(\boldsymbol{\nabla} \times \mathbf{H})\right]+\int_{V} d \tau \mathbf{H} \cdot \boldsymbol{\nabla} \nabla G,
$$


and using again the method outlined between (18) and (20), we may transorm this (using a normal vector $\mathbf{n}$ pointing out of the cone) into:

$$
\mathbf{H}_{s c}^{\prime}=-\int_{S_{b}+S_{01}} d S\left\{(\mathbf{n} \times \mathbf{H}) \times \boldsymbol{\nabla} G+[\mathbf{n} \times(\boldsymbol{\nabla} \times \mathbf{H})] \cdot \mathcal{L}_{\}},\right.
$$

where we introduced Schwinger's [Brown, 1959, and Levine and Schwinger, 1950] tensor Green's function:

$$
\mathscr{G}\left(\mathbf{r}, \mathbf{r}^{\prime}\right)=\left[\mathscr{I}-k^{-2} \nabla \nabla^{\prime}\right] G\left(\mathbf{r}, \mathbf{r}^{\prime}\right),
$$

I being the unit dyadic. We prefer the formulation (A19) to the equivalent one of Stratton and Chu [Stratton, 1941] since it demonstrates directly the transversality of the far field. Indeed, for $r^{\prime} \rightarrow \infty$, we obtain

$$
\mathbf{H}_{s c}^{\prime}(\infty)=i\left(4 \pi r^{\prime}\right)^{-1} e^{i k r} \mathbf{k} \times \int_{S_{01}} d S\{\mathbf{n} \times \mathbf{H}+\hat{k} \times(\mathbf{n} \times \mathbf{E})\} e^{-i \mathbf{k} \cdot \mathbf{r}},
$$

with $\hat{k}=\mathbf{k} / k$ a general direction of scattering. The transversality is obvious. We have here dropped the contribution of $S_{b}$ in the limit of going over to a semi-infinite cone, where it is removed to infinity and gives no contribution as usual.

For backscattering, $\hat{k}=-\mathbf{n}_{0}$, we find in analogy to $(62)$ :

with $\mathbf{J}_{2}$ as before, and

$$
\mathbf{H}_{b s c}^{\prime}(\infty)=-i k\left(4 \pi r^{\prime}\right)^{-1} e^{i k r^{\prime}}\left(\mathbf{J}_{2}+\mathbf{J}_{5}\right) \text {, }
$$

$$
\mathbf{J}_{5}=\int_{S_{01}} d S \mathbf{n}_{0} \times(\mathbf{n} \times \mathbf{H}) e^{i \mathbf{k}_{0} \cdot \mathbf{r}} .
$$

The physical-optics field on the surface of the coating, $S_{01}$, corresponding to nose-on incidence on the cone, is again evaluated with the help of appendix 1, and we obtain

$$
\mathbf{J}_{5}=\left(2 k^{2}\right)^{-1} \pi \mathbf{H}_{0} \tan ^{2} \vartheta \cos (a w) e^{-2 i a \operatorname{esc} \vartheta}\left\{N \sin \vartheta \Delta_{a}^{-1}+w \Delta_{b}^{-1}\right\} .
$$

If the radar cross section is written as in (47), we obtain the simple result $S=\frac{1}{2} e^{-2 i a \csc \vartheta}\left\{\left[N^{2} \sin \vartheta \cos (a w)+i \mu w \sin (a w)\right]\left[N^{2} \sin \vartheta \cos (a w)\right.\right.$

$$
\begin{aligned}
&-i \mu w \sin (a w)]^{-1}+[w \cos (a w)+i \mu \sin \vartheta \sin (a w)] \\
&\left.\cdot[w \cos (a w)-i \mu \sin \vartheta \sin (a w)]^{-1}\right\},
\end{aligned}
$$

and the cross section again goes over into that of a bare perfectly conducting cone (in physicaloptics approximation) if $a \rightarrow 0$ or if $N \rightarrow 1, \mu \rightarrow 1$.

I am grateful to V. H. Weston for many illuminating discussions and encouragements, and to K. M. Siegel for his interest. Helpful discussions with I. Schensted and T. Senior are also acknowledged.

\section{References}

Brown, W. M. (Apr. 1959), An approximation method for large-angle scattering of high-energy scalar and vector waves, Ph. D. Thesis, Department of Physics, University of California, Los Angeles.

Hansen, W. W. and L. I. Schiff (Sept. 1948), Theoretical study of electromagnetic waves scattered from shaped metal surfaces, Microwave Laboratory, Stanford University, Quarterly Report No. 4 (Final Report), Contract W28-009-333.

Hiatt, R. E., K. M. Siegel, and H. Weil (1960), Forward scattering by coated objects illuminated by short wavelength radar. Proc. IRE 48, 1630. 
Hiatt, R. E., K. M. Siegel, and H. Weil (1960), The ineffectiveness of absorbing coatings on conducting objects illuminated by long wavelength radar, Proc. IRE 48, 1636.

Kerr, D. R. (1951), Propagation of short radio waves, Radiation Laboratory Series, 13, p. 454 (McGraw-Hill Book Co., Inc., New York, N.Y.).

Levine, H., and J. Schwinger (1950), On the theory of electromagnetic wave diffraction by an aperture in an infinite plane conducting screen, Comm. Pure App. Math. 3, 355.

Saxon, D. S. (1957), Formulation of high energy potential scattering problems, Phys. Rev. $10 \%, 871$.

Saxon, D. S. (1959), Modified WKB methods for the propagation and scattering of electromagnetic waves, IRE Trans. Ant. Prop. AP-y, Special Supplement, S320.

Saxon, D. S., and L. I. Schiff (1957), Theory of high energy potential scattering, Nuovo cimento 6,614 .

Siegel, K. M., and A. Alperin (Jan. 1952), Studies in radar cross section III: Scattering by a cone, Report UMM-87, Willow Run Research Center, The University of Michigan.

Siegel, K. M., J. W. Crispin, and C. E. Schensted (1955), Electromagnetic and acoustical scattering from a semi-infinite cone, J. Appl. Phys. 26, 309.

Sommerfeld, A. (1959), Optik, Akademische Verlagsgesellschaft Leipzig, 2d ed., pp. 178-292.

Stratton, J. A. (1941), Electromagnetic Theory (McGraw-Hill Book Co., Inc., New York, N.Y.).

Überall, H. (1962), Scattering of electromagnetic waves in Saxon-Schiff theory, Phys. Rev. 128, 2429.

Weston, V. H., and R. Hemenger (1962), High frequency scattering from a coated sphere, J. Res. NBS 66D (Radio Prop.), No. 5, 613-619.

Weston, V. H. (private communication). The statement is based on experimental results.

(Paper 68D6-373) 